AperTO - Archivio Istituzionale Open Access dell'Università di Torino

\title{
JEM-EUSO observational technique and exposure
}

\section{This is a pre print version of the following article:}

Original Citation:

\section{Availability:}

This version is available http://hdl.handle.net/2318/1636722

since 2017-05-19T18:33:59Z

Published version:

DOI:10.1007/s10686-014-9376-3

Terms of use:

Open Access

Anyone can freely access the full text of works made available as "Open Access". Works made available under a Creative Commons license can be used according to the terms and conditions of said license. Use of all other works requires consent of the right holder (author or publisher) if not exempted from copyright protection by the applicable law. 


\title{
The JEM-EUSO observational technique and exposure
}

\section{The JEM-EUSO Collaboration}

Received: date / Accepted: date

\begin{abstract}
In this paper we describe the observational principle and the expected performances of JEM-EUSO. Designed as the first mission to explore the ultra-high energy universe from space, JEM-EUSO monitors the Earth's atmosphere at night to record the UV (300-430 nm) tracks generated by the Extensive Air Showers. We present the expected geometrical aperture and annual exposure in nadir and tilt modes for Ultra-High Energy Cosmic Rays as a function of the ISS altitude.
\end{abstract}

Keywords JEM-EUSO · Ultra-High Energy Cosmic Rays · Extensive Air Showers

\section{Introduction}

JEM-EUSO (Extreme Universe Space Observatory on-board Japanese Experiment Module) [1,2] consists of a UV telescope and an atmosphere monitoring system. Orbiting the Earth every $\sim 90$ minutes at an altitude of $H_{0} \sim 400 \mathrm{~km}$, JEM-EUSO

M. Bertaina (corr. author)

Dipartimento di Fisica, Università di Torino \& INFN Torino, via P. Giuria 1, I-10125 Torino, Italy

Tel.: +39-011-6707492

Fax: +39-011-6707493

E-mail: bertaina@to.infn.it

P. Bobik (corr. author)

Department of Space Physics, Institute of Experimental Physics, Kosice, Watsonova 47, SK-040 01 Kocise, Slovakia

E-mail: bobik@saske.sk

F. Fenu (corr. author)

Institute for Astronomy and Astrophysics, Kepler Center, University of Tübingen, Sand 1, D-72076

Tübingen,Germany

E-mail: francesco.fenu@astro.uni-tuebingen.de

K. Shinozaki (corr. author)

Institute for Astronomy and Astrophysics, Kepler Center, University of Tübingen, Sand 1, D-72076

Tübingen,Germany

E-mail: kenjikry@riken.jp 
is designed to detect UV (300-430 nm) fluorescence photons produced along the track of Extensive Air Shower (EAS) in the atmosphere. The main telescope, which contains a wide Field-of-View ( $\sim 0.85 \mathrm{sr}$; FoV) optics composed by Fresnel lenses, records the EAS-induced tracks with a time resolution of $2.5 \mu$ s (Gate Time Unit; GTU). The Focal Surface (FS) detector is formed by 137 Photo Detector Modules (PDMs) composed by $\sim 5000$ Multi-Anode Photo Mutiplier Tubes (MAPMTs) in total. The FS detector is highly pixelzed by $\sim 3 \times 10^{5}$ channels serving a spatial resolution of $\sim 0.075^{\circ}$, equivalent to $\sim 0.5 \mathrm{~km}$ at ground seen by an altitude of $\sim 400 \mathrm{~km}$. These time-segmented images allow an accurate measurement of the energy and arrival direction of the primary particles.

Since the ISS orbits the Earth in the latitude range $\pm 51.6^{\circ}$, moving at a subsatellite speed of $\sim 7 \mathrm{~km} \mathrm{~s}^{-1}$, the variability of the FoV observed by JEM-EUSO is much higher than that observed by ground-based experiments. In particular, the atmospheric conditions, which eventually determine the aperture, must be carefully monitored via an atmosphere monitoring system consisting of an infrared camera and a steerable laser [3] .

In the following, we describe fundamental principles of JEM-EUSO including the peculiarity of the space-based observation of EASs. We also discuss key parameters relevant to the performance of the JEM-EUSO mission. In this work, we evalute the geometrical aperture and expected exposure in various observational conditions.

\section{Observational principle}

The space-based observation of Ultra-High Energy Cosmic Rays (UHECR) has some peculiarities in comparison to ground-based measurements. Above all, by observing from $\sim 400 \mathrm{~km}$ orbit, JEM-EUSO significantly enhances the aperture compared to any existing and planned observatories. In addition to look vertically down to the nadir (nadir mode), the JEM-EUSO telescope may be tilted astern (tilt mode) increasing the observation area to explore much rare events at highest energies. Thanks to the ISS orbit, JEM-EUSO surveys both hemispheres in Celestial Sphere with a rather uniform exposure, minimizing the systematic uncertainties. This property is distict from ground-based observatories that may only observe different parts of the sky.

There are other interesting aspects in using space-based UHECR observatories. First, the almost constant distance between the detector to the EAS considerably reduces problems associated with the determination of the solid angle and with the different attenuation suffered by the UV light in the atmosphere. Second, the nearly constant fluorescence yield simplifies the reconstruction of the EAS development profile in atmosphere. Third, the observation from space eliminates uncertainties due to scattering by aerosols mostly concentrated near the planetary boundary layer. Finally, as the EAS maximum occurs, for most zenith angles, at altitudes higher than $\sim 3 \mathrm{~km}$ from the Earth's surface, space-based measurements are also possible in cloudy conditions. Compared to ground-based detectors, the observational duty cycle is mainly limited by the moon phases, while the cloud impact is less relevant. 
The JEM-EUSO observational approach mainly relies on the fact that a substantial fraction of the UV fluorescence light generated by the EAS can reach a light-collecting device of several square meters located at several hundreds kilometers away. Typically several thousands photons reach the JEM-EUSO detector for a shower produced by a $10^{20} \mathrm{eV}$ particle. JEM-EUSO is designed to record not only the number of photons but also their direction and arrival time. It allows the measurement of the specific space-time correlation of the signal that helps identifying EAS tracks very precisely in the night glow background.

In order to investigate the shower properties and the detector response we employed the software package ESAF (Euso Simulation and Analysis Framework) [4]. Details of the shower simulation as well as the detector and reconstruction performances are described in [5-7].

Fig. 1 [5], top panel shows the projected tracks on the Earth's surface for EASs with $E=10^{20} \mathrm{eV}$ and zenith angles $\Theta$ of a) $30^{\circ}$, b) $60^{\circ}$ and c) $75^{\circ}$ along with the map for the entire FoV in the sub-panel. Bottom panel shows the image on the FS detector for the case b) in which the integrated counts for each pixel are indicated. The regions enclosed by thick dashed lines in both panels refer to the same PDM.

Fig. 2 shows the arrival time distribution of photons at the telescope entrance aperture from the EASs shown in Fig. 1. The shaded histogram is for $\Theta=60^{\circ}$ and those with solid and dashed lines are for $\Theta=75^{\circ}$ and $30^{\circ}$, respectively.

Up to zenith angles $\sim 60^{\circ}$, the EAS is fully contained within an FoV equivalent to one PDM. It reaches two PDMs around $\Theta \sim 75^{\circ}$. This is the reason that the trigger architecture is based on the PDM scale. The typical FoV of a PDM for $H_{0}=400 \mathrm{~km}$ is about $30 \mathrm{~km}$ on a side $\left(\sim 1000 \mathrm{~km}^{2}\right)$. This means that the entire FS detector can be considered as the sum of 137 quasi-independent sub-detectors corresponding to PDMs. This is important for evaluating the effects of clouds and city light. It should be mentioned here that when a trigger is issued on a PDM, the data of the neighboring PDMs are also retrieved. Another important consideration is that more inclined EASs give brighter signals at EAS maximum and total integrated light. This can be used to extend the energy range of measurement to lower energies by simple geometrical cuts. Moreover, inclined showers allow almost fully calorimetric measurement of the EAS because the entire profile is visible. This is generally not the case of groundbased detectors, which typically select events up to $60^{\circ}$ in zenith angles, and for which the EAS is truncated at ground in many cases.

\section{Observational duty cycle, local light effects and the role of clouds}

The estimation of the exposure of a space-based experiment such as JEM-EUSO requires accounting for: a) the characteristics of the EAS development in the atmosphere as observed from space, $b$ ) the properties of the telescope, including its orbit and $\mathrm{FoV}, \mathrm{c}$ ) the various sources of steady background like night-glow and moonlight, d) the overall optical transmission properties of the atmosphere, in particular the possible presence of clouds, and e) the effect of anthropogenic light, atmospheric flashes such as transient luminous events and meteors. Items a) and b) are the principal factors determining the threshold in energy and maximum aperture of the telescope. 

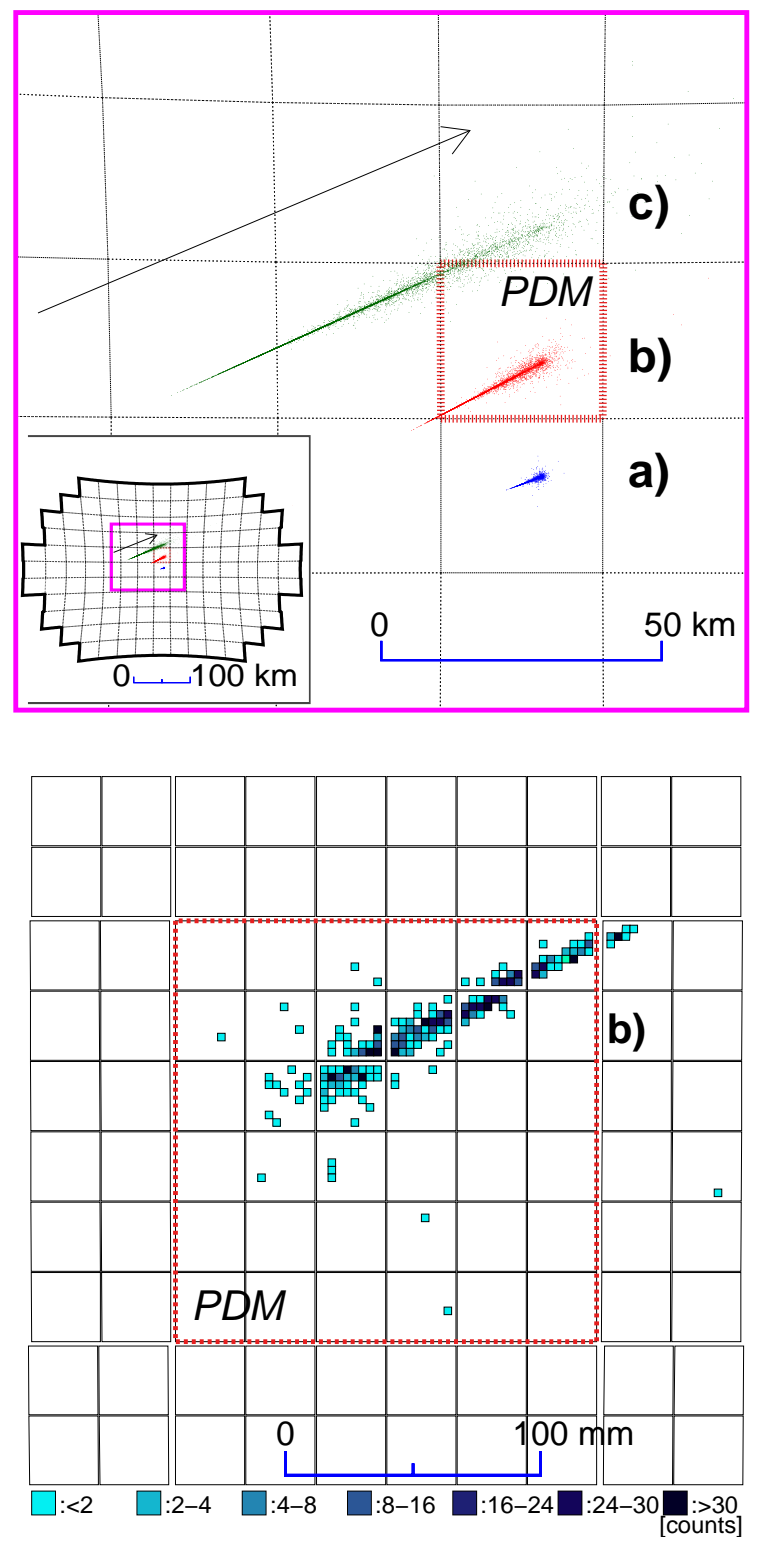

Fig. 1 Top panel shows the projected tracks on the Earth's surface for EASs with $E=10^{20} \mathrm{eV}$ and zenith angles $\Theta$ of a) $30^{\circ}$, b) $60^{\circ}$ and c) $75^{\circ}$. The dashed lines indicate the corresponding areas for the FoV of individual PDMs. In the sub-panel, the corresponding area of the plot is represented by solid lines within the entire FoV. Bottom panel shows the image on the FS detector for the case b). The large squares denotes MAPMTs. The matrix of pixels are indicated with the integrate counts in discrete scale. The regions enclosed by thick dashed lines in both panels refer to the same PDM. Panels herein are quoted from Ref. [5] 


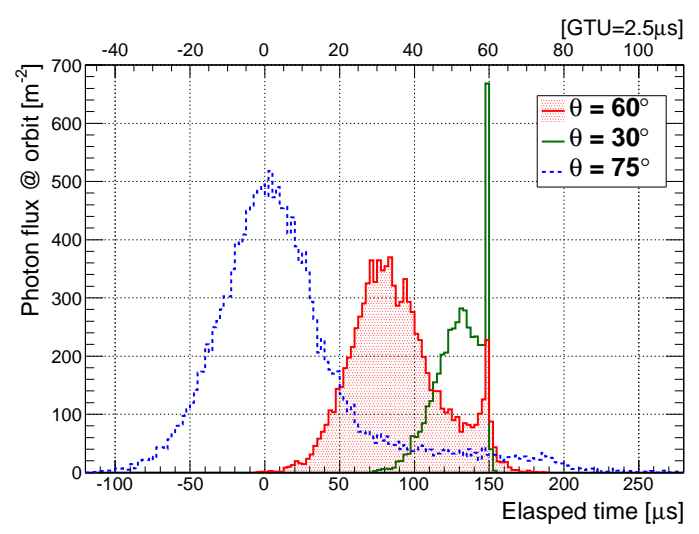

Fig. 2 Arrival time distribution of photons at the telescope entrance aperture from the same EASs shown in Fig. 1 [5]. Shaded histogram denotes the case of $\Theta=60^{\circ}$ and those with solid and dashed lines are for the cases of $\Theta=75^{\circ}$ and $\Theta=30^{\circ}$, respectively.

Item c) limits the observational duty cycle of the mission. Items d) and e) affect the instantaneous aperture of the telescope.

All these above points have been extensively studied in Ref. [5]. We report here the main conclusions. The estimation indicates that the operational duty cycle of JEM-EUSO, or the fraction of time in which the EAS measurement is not hampered by the brightness of the atmosphere, is of the order of $\eta_{0} \sim 20 \%$. This value is conservative at energies $\mathrm{E}>10^{20} \mathrm{eV}$ where it is possible to operate also in higher background levels. The local light such as city light, atmospheric flashes and auroras reduce the effective instantaneous observational area to $1-f_{\text {loc }} \sim 90 \%$ of the geometrical area. The role of clouds has been thoroughly investigated and the cloud efficiency, defined as the ratio of the effective average aperture to the geometrical aperture, is found to be $\kappa_{c} \sim 72 \%$. All the above factors give an overall conversion factor from geometrical aperture to exposure of about $\sim 13 \%$.

\section{Geometrical aperture in nadir mode}

A detailed study of the geomerical aperture as a function of energy for the standard assumption of $400 \mathrm{~km}$ ISS altitude and nadir view with proton injected primaries is extensively described in Ref. [5]. Here we report only the main results and we refer to that analysis for the details of the technique.

To estimate the geometrical aperture, a large number of EASs are simulated by uniformly injecting them over an extended area $S_{\text {inject }} \gg S_{\text {obs }}$ in a clear atmosphere condition for nominal background level of $I_{\mathrm{BG}}=500$ photons $\mathrm{m}^{-2} \mathrm{sr}^{-1} \mathrm{~ns}^{-1}$.

For $N_{\text {trig }}$, triggering samples among $N_{\text {inject }}$, simulated EAS events with an energy $E$, the corresponding geometrical aperture $A(E)$ is defined by the following relation:

$$
A(E)=\frac{N_{\text {trig }}}{N_{\text {inject }}} \cdot S_{\text {inject }} \cdot \Omega_{0},
$$




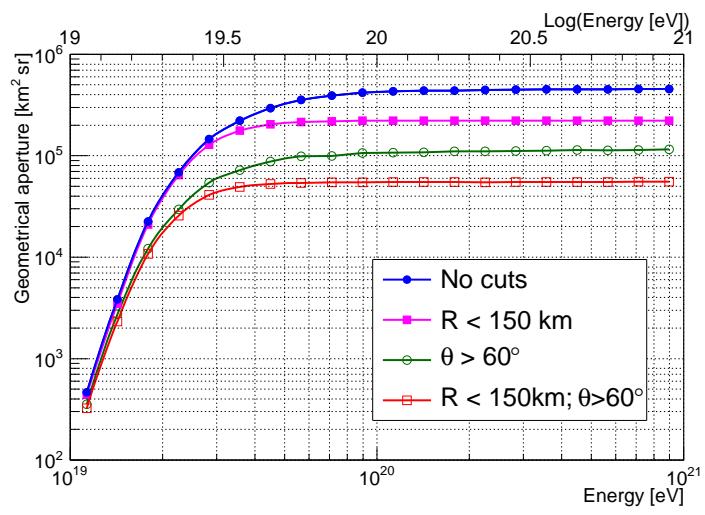

Fig. 3 Geometrical aperture as a function of energy. The filled circles and squares indicate geometrical apertures for the entire observation area and $R<150 \mathrm{~km}$ respectively, where $R$ indicates the distance of the impact location of the EAS from the center of FoV. The open circles and squares include a zenith angle cut of $\Theta>60^{\circ}$.

where $\Omega_{0}=\pi \mathrm{sr}$ is the solid angle acceptance for $0^{\circ} \leqslant \Theta \leqslant 90^{\circ}$. As explained before, by applying simple cuts on the distance $R$ from the center of FoV to the impact location of the EAS, and on the lower limit $\Theta_{\text {cut }}$, the geometrical aperture $A_{\text {sub }}$ is derived as follows:

$$
A_{\text {sub }}(E)=2 \pi \int_{S_{\text {sub }}} \int_{\Theta_{\text {cut }}}^{\pi} \varepsilon(E, \Theta, \mathbf{r}) \cdot \cos \Theta \cdot \sin \Theta d \Theta d S,
$$

where $d S$ is the area element in the selected subsection of the observation area $S_{\text {sub }}$, and $\varepsilon(E, \Theta, \mathbf{r})$ is the probability of trigger at the impact location $\mathbf{r}$ with respect to the center of FoV.

Fig. 3 shows the geometrical aperture as a function of energy for $H_{0}=400 \mathrm{~km}$ along with the apertures for different geometrical cuts in $\Theta$ and $R$.

The geometrical aperture without geometrical cut reaches the plateau ${ }^{1}$ above $\sim(6-7) \times 10^{19} \mathrm{eV}$. At the highest energies, the geometrical aperture is close to saturation. The value is mainly determined by $S_{\text {obs }}$ for a given $H_{0}$ and, therefore, higher altitudes result in larger saturating apertures. Due to a minor contribution of EAS crossing the FoV, the geometrical aperture grows slightly with energy.

By applying the cut $\Theta>60^{\circ}$, which reduces the solid angle acceptance to $\pi / 4 \mathrm{sr}$, a constant aperture is achieved above $\sim(4-5) \times 10^{19} \mathrm{eV}$. In addition, a more stringent cut with $R<150 \mathrm{~km}$ extends the constant aperture range down to $\sim 3 \times 10^{19} \mathrm{eV}$. The possibility to extend the plateau region at lower energies for a subset of events allows a cross-check of the flux measured by the full sample of events in the specific range of energies where the aperture of the instrument is not at the plateau level. Consequently, the overlapping energy range between JEM-EUSO and ground-based observatories is enlarged.

\footnotetext{
1 It is defined by the condition in which the geometrical aperture is $>0.8 \cdot S \cdot \Omega$ for the area $S$ and solid angle acceptance $\Omega$ defined by specific geometrical cuts.
} 


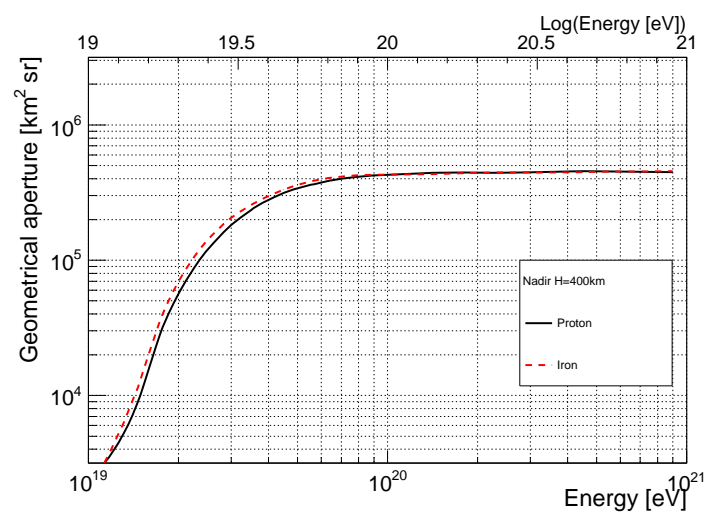

Fig. 4 Aperture as a function of energy at ISS altitude $H_{0}=400 \mathrm{~km}$ without geometrical cuts: solid line for proton and dashed one for iron simulated events.

Fig. 4 shows the comparison between the aperture between proton and iron simulated showers without geometrical cuts. The EAS observation from space has a better visibility of the early stages of the shower development compared to ground-based observation. Iron showers tend to cascade higher in atmosphere compared to proton ones and the apparent length of the EAS before impacting on the Earth's surface or on a cloud top is a bit longer. Simulation results indicate in case of iron showers that a slightly higher number of photons reaches JEM-EUSO in comparison to proton showers with same energy and geometry. This results in a slightly improved trigger efficiency and increased overall exposure in case of iron showers. In the following we will refer always to the results obtained with proton simulated showers.

\section{Geometrical aperture in tilt mode: preliminary results}

An interesting option for JEM-EUSO is the possibility of tilting the telescope. In the tilt mode, the observation area is scaled by $\propto(\cos \xi)^{-3}$ as a function of titling angle $\xi$ of the optical axis from the nadir. This increases the sample of events at the highest energies and helps to compensate the reduction of the observation area in case of periods of lower orbiting altitudes. However, the larger distance under which showers appear make them to be dimmer. For this reason the advantages of the tilt mode are not obvious by themselves and the improvement in exposure depends on the energy in a non trivial way. Therefore, a devoted study must be performed to find a reasonable trade-off between increasing the exposure and keeping the threshold in energy at an acceptable level.

In the present study a set of different tilting angles between $20^{\circ}$ and $40^{\circ}$ has been simulated and compared to the nadir mode and first results obtained. It is important to underline at this stage that the analyses in which the optical axis is tilted by $\sim$ $0^{\circ}-25^{\circ}$, can be easily assimilated to the nadir one (defined in the following 'quasinadir mode'). In case of even larger tilting angles ( $\xi \gtrsim 25^{\circ}$, tilt mode), a dedicated 


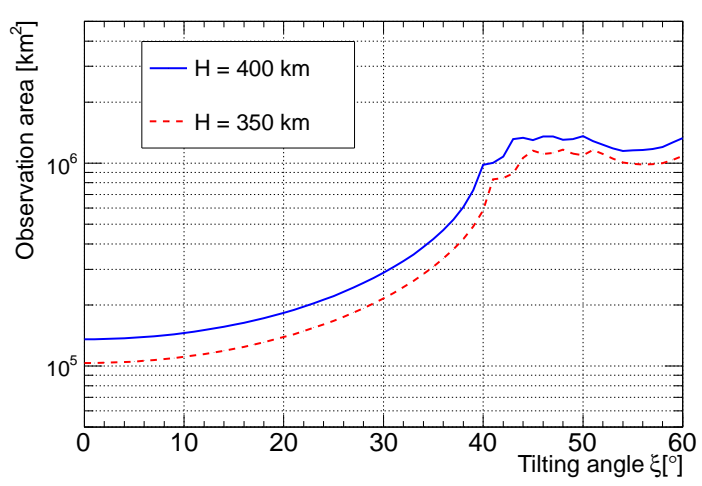

Fig. 5 The JEM-EUSO observation area are shown as a function of the tilting angle for ISS altitude 400 $\mathrm{km}$ and $350 \mathrm{~km}$. The tilting angle $\left(\xi \sim 40^{\circ}\right)$ at which the edge of the FoV reaches the horizon is indicated as well.

study is necessary to evaluate the performance in terms of quality of the reconstructed events. This will be addressed in future, in particular for the $\xi=40^{\circ}$ case where the FoV increases significantly and reaches the horizon. However, we report in the following first studies based on the trigger events for both cases: quasi-nadir and tilt modes. They are based on the assumption of a standard ISS height of $400 \mathrm{~km}$, and a more pessimistic case, in which the ISS flies at much lower altitudes $\left(H_{0}=350 \mathrm{~km}\right)$. This second case is meant to indicate that lower altitudes allow lowering the threshold in energy with a wider range of superposition with ground-based detectors, and that the loss in aperture in nadir mode compared to the standard ISS height would be recovered at the highest energies by operating in quasi-nadir mode.

Fig. 5 shows the dependence of the observation area as a function of the tilting angle for $H_{0}=400 \mathrm{~km}$ and $300 \mathrm{~km}$. The area increases with $\xi$ and reach $\sim 6$ times of that of the nadir mode at $\xi=40^{\circ}$ where a part of FoV sees the local horizon.

In order to estimate the aperture, a specific night glow background has to be assumed. In the present work the nominal background level of 500 photons $\mathrm{m}^{-2} \mathrm{sr}^{-1} \mathrm{~ns}^{-1}$ is assumed constant over the entire FoV. Most likely this is a too simplistic assumption since the background radiance depends on the tilting angle under which the atmosphere is observed. However, at a very first approximation and especially for low tilting angles we can consider the shower to detector distance to be the leading factor affecting the threshold in energy. In fact, the shower signal can be expected to decrease proportionally to the inverse square of the distance which is much stronger than any reasonable increase of the background rate as a function of the tilting angle. Moreover, the increase of the background level $I_{\mathrm{BG}}$ corresponds to an increase of the threshold in energy proportional to $\sqrt{I_{\mathrm{BG}}}$. On the other hand the signal variations affect linearly the threshold. Efforts are going on for a more careful estimation of the background dependence as a function of the tilting angle.

Fig. 6 shows the aperture as a function of energy for different tilting angles. The ISS altitude $H_{0}=400 \mathrm{~km}$ and no geometrical cuts are assumed. As expected by 


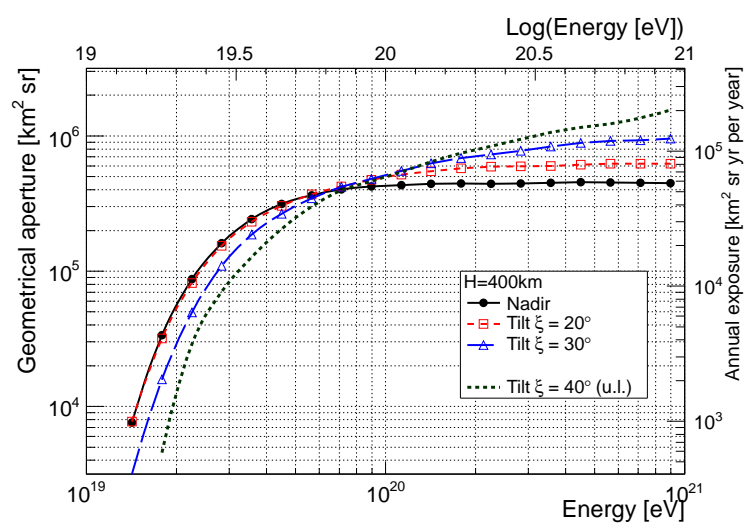

Fig. 6 The JEM-EUSO aperture as a function of energy for different tilting angles. $H_{0}=400 \mathrm{~km}$ and no geometrical cuts are assumed. On the right, the corresponding yearly growth in exposure is shown (see Section 6). In case of $\xi=40^{\circ}$ case, the dashed line shows the upper limit (u.1.) on the reachable aperture and exposure.

tilting the telescope the threshold in energy increases as well as the aperture at the highest energies. The quasi-nadir configuration of $\xi=20^{\circ}$ allows to keep almost constant aperture at the lowest energies, while increasing it moderately (10\%-20\%) at $E>10^{20} \mathrm{eV}$. The tilt mode is suitable to increase the aperture at the energies $E \gtrsim 2 \times 10^{20} \mathrm{eV}$ where the flux is particularly low, reaching a factor of $\sim 1.8$ higher aperture at $E>5 \times 10^{20} \mathrm{eV}$ for the $\xi=30^{\circ}$ case. On the right, the corresponding annual exposure is shown (see Section 6 for discussion).

The tilt mode shows a more slowly growing aperture compared to the nadir or quasi-nadir mode. This is due to the fact that with increasing the energy the more distant PDMs start being more efficient in detecting EAS. During the data taking in the mission, the proper understanding of the growth of the aperture as a function of energy will be verified experimentally by comparing the measured flux in nadir or quasi-nadir mode in the energy range where both tilt and nadir modes provide comparable statistics $\left(5 \times 10^{19} \mathrm{eV}<E<3 \times 10^{20} \mathrm{eV}\right)$.

Fig. 7 shows JEM-EUSO aperture as a function of energy in nadir mode $\left(H_{0}=\right.$ $400 \mathrm{~km}$ and $350 \mathrm{~km})$ as well as the aperture in quasi-nadir mode $\left(\xi=25^{\circ}\right)$ for $H_{0}=$ $350 \mathrm{~km}$. At the lowest energies the highest trigger efficiency of the $H_{0}=350 \mathrm{~km}$ allows to guarantee the same aperture of the standard configuration. Above $10^{20} \mathrm{eV}$ the aperture can be recovered by the quasi-nadir configuration. Only a moderate loss of aperture (below 20\%) in the range $3 \times 10^{19} \mathrm{eV}<E<10^{20} \mathrm{eV}$ remains. However, this is the region where the acquired statistics is relatively high.

\section{Exposure}

From the above results, the exposure per year of operation for events that trigger JEM-EUSO, defined as the 'annual exposure' is evaluated as a function of energy:

$$
(\text { Annual exposure }) \equiv A(E) \cdot \kappa_{\mathrm{C}} \cdot \eta_{0} \cdot\left(1-f_{\mathrm{loc}}\right) \cdot(1[\mathrm{yr}]) .
$$




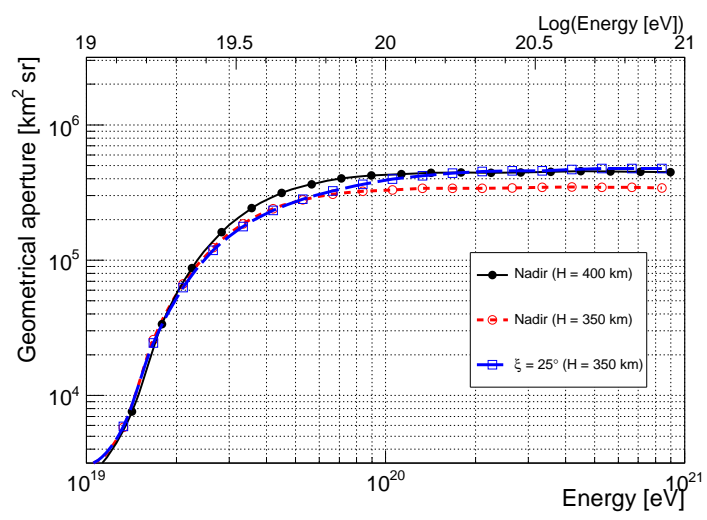

Fig. 7 The JEM-EUSO aperture as a function of energy for nadir mode at $H_{0}=400 \mathrm{~km}$ and $350 \mathrm{~km}$ as well as that for quasi-nadir mode $\left(\xi=25^{\circ}\right)$ at $H_{0}=350 \mathrm{~km}$. All plots refer to the one without geometrical cuts.

In this estimation, we use $\kappa_{\mathrm{C}}=72 \%, \eta_{0}=20 \%$, and $f_{\text {loc }}=10 \%$, respectively and independently of the tilting angle. In reality, it is expected that the cloud inefficiency will be lower for the tilting mode. This is due to the fact that the PDMs looking at farther distances are more efficient in detecting inclined showers that develop in higher atmospheric levels and deliver more light to the telescope (see Fig. 2). Therefore, the cloud inefficiency is less important. On the other hand the effect of local light sources will be more pronounced because the FoV of such PDMs is larger. These two factors compensate each other at a first approximation, however, a dedicate study will be conducted in future to estimate the second order effects. The operational inefficiencies related to ISS (rockets docking on ISS, lid operation, detector maintenance or aging, etc.) are not taken into account yet. The fraction of the triggered events that are succesfully reconstructed and the quality of such events is described in detail in $[6,7]$. The present results constitute an upper limit on the effective exposure of the instrument for the assumed conditions.

On the right axis of Fig. 6, the scale for the annual exposure is shown for the geomerical apertures indicated in the figure. For the nadir mode, the JEM-EUSO annual exposure without geometrical cuts is expected to be $\sim 9$ times larger than that of the Pierre Auger Observatory. In tilt mode, the exposure further increases at extreme energies by another factor $\sim 2$ allowing to explore the energy range where much fewer events are expected.

Unlike ground-based observatories, the global ISS orbit and better sensitivities for EAS with large zenith angles allows observation of the entire Celestial Sphere. The exposure distribution has only limited anisotropies in declination and right ascension which are due to the different resident time of the ISS as a function of the latitude, to the different twilight time for different latitudes and to local and seasonal dependence of the cloud distribution and local man-made light. 


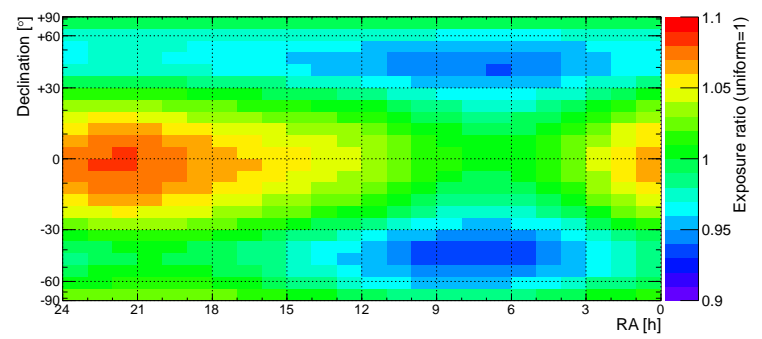

Fig. 8 Expected distribution of observed exposure as a function of declination and right ascension. Clear and cloudy atmosphere are considered.

Fig. 8 summarizes the results when all the above effects are taken into account. The cloud distribution is taken from Ref. [8]. The exposure distribution over Celestial Sphere is rather uniform within $\pm 10 \%$ level.

\section{Conclusions}

Simulations show that JEM-EUSO reaches almost full efficiency in nadir mode already at energies around $3 \times 10^{19} \mathrm{eV}$ for a restricted subset of events, and provides full aperture at energies $E>(6-7) \times 10^{19} \mathrm{eV}$. The expected exposure is essentially independent of the incident primary particle. The observational duty cycle and the role of clouds have been summarized. The expected annual exposure of JEM-EUSO in nadir mode around $10^{20} \mathrm{eV}$ is equivalent to about 9 years exposure of Auger.

The quasi-nadir mode $\left(\xi \lesssim 25^{\circ}\right)$ allows to slightly increase the exposure at $E>$ $10^{20} \mathrm{eV}$. This is an interesting option to recover the exposure from unexpected operational inefficiencies or low ISS altitudes. A first study on tilt mode has been presented. The aperture is expected to increase by a factor $\sim 2$ for a tilting angle $\xi \sim 30^{\circ}$ compared to the nadir mode at the highest energies. However, this result has to be confirmed by further analyses.

Thanks to the ISS orbit JEM-EUSO surveys the entire Celestial Sphere with a very limited non-uniformity of the exposure on declination and right ascension $( \pm 10 \%)$ taking into account coverage distribution and seasonal variation of the clouds.

\section{Acknowledgments}

This work was partially supported by Basic Science Interdisciplinary Research Projects of RIKEN and JSPS KAKENHI Grant (22340063, 23340081, and 24244042), by the Italian Ministry of Foreign Affairs, General Direction for the Cultural Promotion and Cooperation, by the Deutsches Zentrum fuer Luft- und Raumfahrt, by the 'Helmholtz Alliance for Astroparticle Physics HAP' funded by the Initiative and Networking Fund of the Helmholtz Association (Germany), and by Slovak Academy of Sciences MVTS JEM-EUSO as well as VEGA grant agency project 2/0076/13. 


\section{References}

1. Takahashi, Y. et al. (JEM-EUSO Coll.), The JEM-EUSO Mission, New Journal of Physics 11, 065009/1-21 (2009).

2. Adams Jr., J.H. et al. (JEM-EUSO Coll.) An overview of the JEM-EUSO instrument, This volume.

3. Adams Jr., J.H. et al. (JEM-EUSO Coll.) The AM system of the JEM-EUSO instrument, This volume.

4. Berat, C. et al., Full simulation of space-based extensive air showers detectors with ESAF, Astroparticle Physics 33/4, 221-247 (2010).

5. Adams Jr., J.H. et al. (JEM-EUSO Coll.), An evaluation of the exposure in nadir observation of the JEM-EUSO mission, Astroparticle Physics, 44, 76-90 (2013).

6. Adams Jr., J.H. et al. (JEM-EUSO Coll.), Performances of JEM-EUSO: angular reconstruction, This volume.

7. Adams Jr., J.H. et al. (JEM-EUSO Coll.), Performances of JEM-EUSO: energy and $\mathrm{X}_{\max }$ reconstruction, This volume.

8. Adams Jr., J.H. et al. (JEM-EUSO Coll.), The impact of clouds in the JEM-EUSO observations, This volume. 